Cinémas

Revue d'études cinématographiques

Journal of Film Studies

\title{
William Maxwell Aitken, père tout-puissant du cinéma canadien
}

\section{Germain Lacasse}

Volume 10, numéro 1, automne 1999

Cinélekta 3

URI : https://id.erudit.org/iderudit/024801ar

DOI : https://doi.org/10.7202/024801ar

Aller au sommaire du numéro

Éditeur(s)

Cinémas

ISSN

1181-6945 (imprimé)

1705-6500 (numérique)

Découvrir la revue

Citer cet article

Lacasse, G. (1999). William Maxwell Aitken, père tout-puissant du cinéma canadien. Cinémas, 10(1), 13-35. https://doi.org/10.7202/024801ar

\section{Résumé de l'article}

Le cinéma documentaire canadien, principalement produit par l'Office national du film du Canada (ONF), est un cinéma de propagande valorisant le modèle britannique du capitalisme. Ce cinéma a été pensé et organisé pendant la Première Guerre mondiale par William Maxwell Aitken, Lord Beaverbrook, qui décupla la production de films pour stimuler le patriotisme canadien. 


\title{
William Maxwell Aitken, père tout-puissant du cinéma canadien ${ }^{1}$
}

\section{Germain Lacasse}

\begin{abstract}
RÉSUMÉ
Le cinéma documentaire canadien, principalement produit par l'Office national du film du Canada (ONF), est un cinéma de propagande valorisant le modèle britannique du capitalisme. Ce cinéma a été pensé et organisé pendant la Première Guerre mondiale par William Maxwell Aitken, Lord Beaverbrook, qui décupla la production de films pour stimuler le patriotisme canadien.
\end{abstract}

\section{ABSTRACT}

Canadian documentary cinema, produced mostly by the National Film Board of Canada (NFB), is a cinema of propaganda which advocates the British model of capitalism. This cinema was thought out and organized during the First World War by William Maxwell Aitken, Lord Beaverbrook, who increased film production tenfold to stimulate Canadian patriotism.

Les Canadiens croient généralement que la politique cinématographique de leur gouvernement fut surtout développée pendant la Seconde Guerre mondiale, grâce à l'intervention d'un jeune "génie» du cinéma documentaire, John Grierson. Il faut nuancer un peu cette version des faits, car John Grierson ne fut que le successeur et l'homme de main involontaire de William Maxwell Aitken, Lord Beaverbrook, celui-là même qui orchestra toute la propagande filmique britannique et 
canadienne pendant la Première Guerre mondiale. Leurs conceptions de la propagande n'étaient certes pas les mêmes, mais les visées apparemment plus démocratiques de Grierson convenaient tout aussi bien aux besoins immédiats de l'État canadien que, 25 ans plus tôt, les principes expéditifs d'Aitken et ses films édulcorés ne l'avaient fait. Cela autorise à penser qu'Aitken eut peut-être quelque chose à voir avec l'arrivée de Grierson au Canada en 1939 et dans son licenciement après la guerre, quand le pouvoir qu'il avait réussi à constituer ne convint plus aux dirigeants canadiens. Même sommaire, l'examen des trajectoires du Canadien Aitken et du Britannique Grierson font d'un soupçon initial une hypothèse qui pourra se révéler féconde.

Magnat de la presse, chef d'orchestre de la propagande de guerre canadienne et britannique, et particulièrement de la propagande cinématographique, Aitken fut nommé ministre de l'Information de l'Angleterre en 1918. Il quitta bientôt le ministère (pour des raisons stratégiques dont le détail sera exposé plus loin), qui fut d'ailleurs aboli une fois le conflit résolu. On le consulta encore en 1939 quand le ministère fut remis sur pied pour stimuler le sentiment national non seulement en Angleterre, mais dans tout l'Empire britannique (Evans, 1977, p. 94 et 119). Entre temps, la Conférence impériale de 1926 avait encouragé la création de l'Empire Marketing Board (EMB) afin de promouvoir le commerce dans l'Empire britannique, un but politique que défendait déjà Aitken en 1910 et qu'il appuya à nouveau en menant dans ses journaux une longue campagne qu'il appela "Empire Cruisade» (Chisholm et Davie, p. 279). L'EMB mit sur pied une unité de production de films où fut embauché John Grierson en 1927; Grierson y réalisa plusieurs films remarquables pour le compte du gouvernement anglais. Il devint également en 1938 conseiller de l'Imperial Relations Trust (IRT), et fut envoyé à ce titre au Canada et en Australie afin d'étudier la possible influence du cinéma sur le resserrement des liens de l'Angleterre avec ses dominions (Véronneau, p. 9). Son patron d'alors, Sir Stephen Tallents, avait été lui aussi consulté relativement à la création du ministère de l'Information, et avait sans doute discuté de l'embauche de Grierson avec 
Aitken, dont l'ombre planait sur toute la politique impériale britannique. Il n'est pas établi qu'Aitken intervint directement dans l'envoi de Grierson au Canada. Mais il est certain que l'expérience d'Aitken dans l'exercice et le contrôle de la propagande, son influence directe sur toutes ces questions en Angleterre, son implication dans les innombrables structures officielles et officieuses de l'époque furent autant de ficelles manipulées par ce maître du pouvoir et auxquelles pendait nécessairement Grierson ${ }^{2}$. Pour bien comprendre l'influence et l'importance qu'eut Aitken sur l'histoire canadienne, sa cinématographie de guerre et la carrière de Grierson, il faut remonter à la guerre de 1914-1918, début véritable de l'intervention étatique canadienne dans le monde du cinéma.

\section{La lecture de guerre}

Le gouvernement canadien, qui s'était jusqu'alors peu intéressé au cinéma sinon pour imposer certaines mesures de contrôle et de surveillance, resserre ces mesures dès le début de la guerre: la soumission des films au bureau de censure de la presse devient obligatoire; ce bureau scrute d'ailleurs toute production médiatique. Mais de plus, le gouvernement se lance dans la production de films, soit directement, soit par l'entremise de compagnies privées spécialisées dans la production de films d'actualité. La manière dont le gouvernement et l'armée liront la guerre, ainsi que l'écriture qui en découlera, seront une lecture et une écriture hégémoniques et occultantes. Tandis que les soldats rampaient dans la boue sous des pluies d'obus et vivaient l'expérience technologique la plus dévastatrice de l'histoire, les spectateurs canadiens voyaient des soldats souriants, saluant la caméra et assistant bien rangés aux défilés des dignitaires.

Si l'on peut classer la propagande orchestrée par John Grierson entre 1939 et 1945 dans la catégorie des lectures et institutions "documentarisantes»(Odin, 1984, p. 264), la lecture instituée pour la guerre de 1914-1918 doit être appelée "ciné-journalistique": au lieu de dramatiser les faits et gestes des populations engagées dans la guerre, comme le fera Grierson, Aitken montrait des images d'événements réels, mais dont était bannie toute allusion trop directe aux horreurs de la 
guerre. Les newsreels du conflit étaient d'ailleurs préparés et donnés à lire selon le même dispositif que les journaux appartenant à Aitken, qui menait à travers eux de grande campagnes de revendications au nom des citoyens tout en évitant de décrire trop directement les scandales et d'attaquer de front les responsables. L'historien anglais Luke McKernan a souligné la particularité du newsreel en l'opposant au film d'actualité ou topical d'avant la guerre. Tandis que le topical relatait un événement important dont le récit demeurait digne d'intérêt, le newsreel était "[...] d'un intérêt captivant mais également éphémère, exigeant inlassablement un nouveau matériel de même nature qui imposait sa propre logique" (McKernan, p. 4-8). Ce type de message et de texte était fort similaire à ceux des journaux de masse, développés surtout pendant la même guerre et soumis à une censure aussi sévère. Ils ont créé une pratique de lecture fondée sur la consommation rapide d'anecdotes sans cesse renouvelées. C'est dans ce cadre que la "lecture de guerre" devient elle aussi une lecture anecdotique, s'il l'on s'en tient bien entendu aux textes ou aux films.

Linstitution de ce nouveau genre de textes devait ajuster le cinéma aux conditions imminentes de la guerre moderne. Par sa capacité mimétique et persuasive, le cinéma aurait pu nuire à l'effort de guerre en rendant compte de son horreur. Mais il fut arraisonné dès le début par l'autorité politique qui s'assura de l'élimination soigneuse et sévère de toute trace trop apeurante. Produit emblématique de la modernité et de sa perception médiatisée, le cinéma aurait pu transmettre de façon trop intense l'expérience catastrophique de la guerre moderne où l'immense pouvoir de la technologie est mis au service d'une destruction massive. Mais le cinéma fut dépouillé de ce qu’on pourrait appeler son "attraction réaliste», c'est-à-dire de l'intérêt qu'il suscite par sa capacité à reproduire le réel. Il en fut privé parce que cette capacité aurait probablement dissuadé les populations prêtes à s'enrôler, les convainquant qu'elles allaient droit au carnage. Certains soldats furent sensibles à ce pouvoir du cinéma, ils voyaient dans le dispositif cinématographique une expérience tout à fait comparable à celle du champ de 
bataille (Eksteins, p. 209), dans la mesure où l'image pouvait provoquer des surprises dévastatrices semblables à celles vécues au front. Les spectateurs des quelques rares films réalistes sont là pour en témoigner, eux qui furent frappés de stupeur ou effrayés au point de perdre conscience durant les séances (Chambers, p. 274).

Ce média, qui se déployair au rythme d'un nouveau siècle plein de surprises destructrices, disposé à en montrer toute l'horreur, fut donc détourné vers une culture du vide et du futile, de l'anecdote insignifiante plutôt que de l'histoire. Il faudra attendre un deuxième carnage mondial pour obliger des images à rendre compte de l'horreur inscrite sous les mots progrès et modernité. Nuit et brouillard (Alain Resnais, 1955) fut le premier «documentaire " postmoderne, marquant d'un sceau indélébile la pire et la dernière tragédie de la modernité. Mais il avair été précédé par les derniers newsreels modernes, ceux tournés par les premiers opérateurs américains parvenus à Auschwitz. Ces films étaient, pour le moins, différents des newsreels de la guerre 1914-1918 ou des documentaires de Grierson l'idéaliste. Les films de 1914-1918 étaient en quelque sorte des fictions dont les spectateurs n'étaient dupes, ni les réalisateurs complices. Ces films sont d'assez curieux objets au centre d'un contrat où producteurs et récepteurs savent que la lecture est biaisée et limitée à ces photographies lacunaires. Celles-ci permettent cependant aujourd'hui de comprendre comment en ce début de siècle l'institution lisait la guerre et la donnait à lire.

La "lecture de guerre» est une activité exercée sous de fortes contraintes, une lecture hégémonique. LÉtat-nation accentue le contrôle qu'il exerce sur les institutions et met sur pied ses propres instances de surveillance: bureau de censure et bureau de propagande, organisations de contrôle des textes et d'imposition des discours. Le début de la Première Guerre mondiale marque l'achèvement de l'institutionnalisation du cinéma, enrôlé dans la machine de guerre après avoir joui depuis sa naissance d'une certaine liberté. Non seulement le public connaissait l'existence de ces contraintes, mais il choisissait, selon ses préférences idéologiques ou politiques, d'adhérer à cette lecture 
ou de la mettre en doute. Les Canadiens qui appuyèrent l'effort de guerre savaient bien que les images qu’ils voyaient étaient édulcorées, mais cela ne les empêcha pas d'approuver cette censure et cette lecture biaisée. Ceux qui s'opposèrent à la guerre, ou du moins à l'enrôlement volontaire, critiquèrent probablement cette représentation fictive parce qu'ils n'en approuvaient pas le motif ou la finalité. À chaque fois, une lecture hautement politique s'empara donc de ces films de fiction comme de post-textes plutôt que pré-textes: c'est pourquoi ces films illustrent a posteriori autant les choix politiques de l'institution que, pour une sémio-pragmatique du sens, les choix politiques des spectateurs. Malgré leur «insignifiance", ils savent stimuler les lecteurs réceptifs; par leur opacité, accentuer la résistance des spectateurs critiques. Cette double affirmation donne raison aux spécialistes de la question qui à l'instar de Roger Odin affirment que "[...] ce sont les déterminations externes qui régissent le processus de production de sens du texte" (Odin, 1994, p. 27). Ainsi, le film de guerre ne montre pas la guerre, surtout à cette époque; il ne fait qu'illustrer un discours déjà entendu, ou montrer quel discours l'institution veut laisser entendre: des films muets sur une guerre sans bruit.

\section{Le maître de lecture}

À une lecture dirigée, il faut un directeur de lecture. C'est pourquoi il faut absolument se mettre sur la piste de William Maxwell Aitken. Du coup, on comprendra mieux comment il en vint à assumer le rôle de maître de lecture. Une grande partie des films canadiens et britanniques produits durant la guerre 1914-1918 portent sa "signature". Aitken acquit progressivement la direction de tous les organismes supervisant la propagande militaire britannique et canadienne. Ce pouvoir lui permit de porter un soin tout particulier à la propagande cinématographique, supervisant l'embauche et le travail des opérateurs et s'assurant que la partie canadienne de la propagande filmée soit constamment et distinctement visible. Il ne faut pas hésiter à considérer son activité, rarement étudiée de près, comme la première intervention gouvernementale canadienne en matière de propagande cinématographique, inter- 
vention aussi importante, à cette époque, que celle de John Grierson à l'ONF pendant la Deuxième Guerre mondiale. Le gouvernement canadien ne légiféra jamais sur cette question entre 1914 et 1919 , mais l'immense pouvoir qu'il confia à Maxwell Aitken correspond parfaitement aux volontés politiques de l'oligarchie dirigeant alors le Canada. Poursuivant la politique amorcée avec la création de la Confédération en 1867 , les capitalistes canadiens voulurent alors développer le rôle international du Canada. Ils investirent des capitaux de plus en plus considérables à l'étranger et participèrent à la construction des grands blocs monopolistes qui se partageaient le monde économique et politique. Max Aitken croyait que l'entreprise devait passer par un resserrement des liens entre le Canada et l'Angleterre, par l'instauration de tarifs préférentiels avec l'Empire britannique qui intensifieraient les échanges commerciaux, par l'engagement du Canada dans une guerre qui impliquait déjà l'Angleterre, par l'établissement d'une forte activité de propagande à laquelle l'image animée participerait pleinement ${ }^{3}$.

Jeune Canadien devenu millionnaire en naviguant dans le monde canadien de la finance, propriétaire de journaux à Londres et député au Parlement londonien après son émigration en Angleterre vers 1910, et finalement élevé à la pairie britannique sous le nom de Lord Beaverbrook, William Maxwell Aitken est né en Ontario en 1879. Fils d'un pasteur écossais arrivé au Canada en 1865, il passe son enfance au NouveauBrunswick, où la famille avait déménagé en 1880 . Il poursuit des études de manière un peu erratique, ratant l'examen d'entrée à l'Université Dalhousie, mais il prend goût néanmoins à l'écriture journalistique et à la vente d'assurances. Bientôt, il se lance en affaires et connaît un succès extraordinaire. En quelques années, il devient millionnaire et déménage d'Halifax à Montréal en 1906 pour être plus près du centre canadien des affaires. Sa recette était simple mais relativement nouvelle et il l'appliquait avec une détermination qui justifiait tous les procédés. Cette recette: le monopole. Quand il décidait d'investir dans un secteur industriel, il en rencontrait les principales entreprises et les persuadait de se regrouper pour prospérer au lieu de se faire concurrence. Il fallait évidemment très bien 
connaître les affaires et ses lois, mais surtout être doué d'une extraordinaire force de persuasion. Aitken avait tous ces atouts ou sut les développer plus que quiconque. Il forma des cartels dans plusieurs secteurs industriels canadiens: papier, mines, acier, électricité, construction, fondant pour ce faire la firme Royal Securities qui drainait et redistribuait les capitaux.

Jusque-là, l'industrie canadienne était surtout financée par des capitaux familiaux; Aitken innova en lançant la vente d'actions sur le marché public. Véritable génie de la vente et de la persuasion, il connut ainsi un succès phénoménal. Son renom fut tel que les plus grands financiers canadiens lui confièrent leurs fortunes et firent partie des conseils d'administration qu'il formait. Mais cette recette remarquable était aussi quasi illégale et le força probablement à quitter le pays: en 1910, ses partenaires dans le conglomérat Canada Cement intentèrent une poursuite contre lui, alléguant qu'ils avaient été trompés et escroqués. Non seulement Aitken naviguait-il constamment à la limite des conventions antitrust, déjà très élastiques, mais il semble qu'il n'hésitait pas à éliminer des partenaires pour en favoriser d'autres. Aucune tactique ne semble d'ailleurs l'avoir rebuté, ni en affaires ni plus tard en politique, deux secteurs où il aura aussi à gérer des productions cinématographiques.

En 1910, Aitken part pour l'Angleterre en déclarant qu'il a été séduit par le programme politique de Chamberlain visant la consolidation de l'Empire britannique par l'adoption de politiques préférentielles et réciproques en matière commerciale et militaire ${ }^{4}$. Aitken dit vouloir défendre ce programme — baptisé "Tariff Reform" - qui correspond évidemment assez bien à ses intérêts financiers: ses monopoles canadiens, fondés sur un marché protégé, étaient alors sous la menace d'un pacte de libreéchange proposé par Wilfrid Laurier. L'Empire britannique permettait par contre d'ériger des trusts capables de résister à la concurrence de l'Allemagne et des États-Unis, principaux rivaux de l'Angleterre sur la scène mondiale. Le départ pour l'Angleterre lui permet aussi de se faire oublier au Canada où l'affaire Canada Cement jette beaucoup d'ombre sur sa réputation. Son succès en politique est aussi rapide qu'en affaires. En décembre 1910, grâce à des dépenses publicitaires énormes, affectées principalement à 
un journal quotidien distribué gratuitement, il est élu député en Angleterre; il représente le comté d'Ashton-Under-Lyne sous la bannière du parti conservateur bientôt dirigé par Bonar Law dont il devient vite l'ami et l'homme de confiance (Bonar Law est né au Nouveau-Brunswick). Aitken devint aussi rapidement un grand ami de l'écrivain Rudyard Kipling, prophète et chantre de ce renouveau de l'impérialisme britanniques. Aitken accède même à la pairie de l'empire, recevant le titre de Lord Beaverbrook en 1916. La rapidité fulgurante de son ascension dans un monde aussi cloisonné que l'oligarchie financière britannique montre bien sa finesse autant que son audace.

Profitant de ses relations politiques qui lui confient leurs capitaux, il poursuit sa carrière de financier, mais en s'intéressant particulièrement au monde de l'information. Il tente d'abord d'acquérir le Montreal Gazette et le Montreal Daily Star, mais l'entreprise se heurte à l'opposition de Montréalais que les procédés d'Aitken ont peut-être rendus méfiants. En 1911, il achète le Globe, quotidien londonien qu'il revend rapidement faute de pouvoir le transformer. Dès le début de la guerre, il devient responsable de la propagande canadienne et ensuite de la propagande anglaise, démontrant son efficacité aussi rapidement que dans les affaires et la politique. Il se tourne alors résolument vers le commerce des journaux. Graduellement, il acquiert une bonne partie des actions du Daily Express de Londres, qu'il achète en décembre 1916 en partie pour soutenir Bonar Law et la politique impériale. Dès la fin de la guerre, il lancera le Sunday Express, puis l'Evening Standard en 1923 et le Scottish Daily Express en 1928 ; de plus, il achètera des actions du Glasgow Evening Citizen et du Daily Mail, son principal concurrent londonien, tout en continuant d'investir dans d'autres secteurs et de faire fructifier sa fortune. Notons qu'Aitken confie la direction de ses journaux à des Canadiens: l'administration revient à E.-J. Robertson et la direction éditoriale du Daily Express, à Beverley Baxter.

On commence maintenant à voir comment Aitken conjugua ces trois termes: guerre, média et finance. Son intérêt pour le cinéma et les journaux semble lui être venu d'un besoin d'instruments capables de légitimer des placements fondés sur un 
opportunisme parfois cynique. Quelques jours avant le début de la guerre, dans une lettre à Kipling, il s'exprimait ainsi: "If we are to have a European war the advantage to Canada and America will be enormous. The price of wheat will go up and America will have a period of great prosperity" (cité dans Chisholm et Davie, p. 122). Il était bien placé pour le savoir puisqu'en 1912, lui et son ami d'enfance R. B. Bennett avaient acheté une minoterie et cent élévateurs à grain dans l'Ouest canadien. Aitken avait de toute évidence besoin d'une opinion publique supportant la guerre; s'il ne voulait pas la guerre, il avait néanmoins pris certains moyens pour en tirer parti le cas échéant. Son poste de publiciste canadien de la guerre le lança dans l'expérience du journalisme, dont il fit son principal gagnepain en même temps qu'un formidable moyen de contrôle de l'opinion publique. Un peu mis de côté par la classe politique britannique, méfiante envers son audace, son habileté et sa rapidité, il se tourna en quelque sorte vers les journaux pour ajouter au pouvoir que lui donnaient les contacts politiques fondés sur ses lucratifs conseils financiers. Il semble aussi avoir misé sur les journaux pour détenir en temps de paix le pouvoir que lui procurait la propagande pendant la guerre. Il déclara souvent avoir d'abord acheté des journaux pour s'assurer une prise sur l’opinion publique. Il lui fallut d'ailleurs quelques années avant de rentabiliser le Daily Express (Howard, p. 64).

C'est pendant la Grande Guerre qu'en Angleterre les journaux évoluèrent du journal d'opinion vers la grande presse de masse; c'est pendant la Grande Guerre aussi qu'Aitken avait fait ses classes de propagandiste. Le propriétaire de journaux n'avait plus qu'à transférer son savoir. Aitken se disait le défenseur d'une démocratie où chacun avait sa chance et où ses journaux étaient «la tribune du peuple»:

The Daily Express is an independant paper attached to the interests of no party. It is opposed to privilege in any class of the community. It stands for freedom and equality of opportunity for all men. It believes in the abolition in peacetime of the Government's controls brought in by the war. It will uphold the right of the people to advance their own interests and shape their 
own lives, and will oppose all attempts to interfere with the simple and healthy pleasures of the nation (cité dans Chisholm et Davie, p. 209).

Pour défendre ce principe, Aitken lança dans les journaux de grandes campagnes populistes dénonçant à l'occasion les profits honteux tirés de la guerre par certains financiers! Ses journaux étaient surtout consacrés à la nouvelle, mais la factualité n'empêcha jamais une sélection et un traitement des sujets favorables à des revendications précises comme au moment de réclamer ou de défendre les droits du citoyen moyen. John Beavan, éditeur du journal rival Daily Herald, écrivit que les journaux d'Aitken présentaient un grand intérêt sociologique parce qu'ils étaient le meilleur exemple de journaux de masse, populaires dans toutes les classes de la société. En d'autres termes, Aitken savait trouver les sujets et les propos permettant de convaincre chacun de l'équité du capitalisme britannique, comme pendant la guerre il avait su trouver les images persuadant la majorité de l'importance et de la moralité de cette guerre.

Aitken fut l'un des financiers les plus puissants de ce siècle, indubitablement, mais aussi un des principaux concepteurs ou manipulateurs de "l'opinion publique». Il deviendra rapidement le magnat de la presse britannique, poussant le succès de ses journaux à des limites phénoménales, faisant en Angleterre ce que Hearst avait fait aux États-Unis. Le Daily Express vendait 230000 copies quand il l'acheta, et ce chiffre atteignait 4,5 millions à sa mort en 1964 . Il répéta dans le commerce des journaux le fabuleux succès qui avait été le sien dans la vente de titres boursiers. En un certain sens, le cinéma lui servit de banc d'essai pour ses théories sur l'information et la propagande, puisque avant de se lancer dans la presse quotidienne, il sera surtout actif dans la propagande filmée. Les journaux d'Aitken représentent-ils la version pacifique de sa propagande en temps de guerre? Le fait divers représente-t-il la vie en temps de paix autant que le défilé militaire représente la réalité de la guerre? Ses journaux furent-ils aussi nécessaires à ses usines de ciment que les films de guerre le furent pour ses élévateurs à grain? Cette relation du signe au référent est-elle arbitraire? Fascinantes questions, trop vastes et précoces pour être abordées 
avant de parler de l'activité de propagande cinématographique d'Aitken.

\section{Une lecture dirigée}

Le citoyen Lord Beaverbrook fut chargé de la propagande du gouvernement et de l'armée canadienne, de la collecte des archives et de l'histoire militaire, en plus de diriger, en sous-main, toute la propagande britannique". C'est lui qui organisa la lecture de cette guerre, et sa mémoire. Il acquit là un pouvoir aussi rapide et aussi étendu qu'en politique et dans le monde financier. Nous ne discuterons pas rétrospectivement de la légitimité de ce pouvoir; disons simplement qu'il étonne dans une société dite démocratique, engagée dans un soi-disant combat pour préserver la liberté du citoyen. Aitken fut d'abord nommé à un poste qu'il contribua probablement à créer - il avait accouru au Canada pour négocier ce poste dès le début du conflit, en octobre 1914 — :Canadian Eye Witness", c'est-àdire publiciste de la guerre, responsable de la rédaction d'articles relatant l'intervention des troupes canadiennes. Dès le début, il centralisa donc le contrôle des discours relatifs à la guerre. En mars 1915, tandis que l'accès au front était encore interdit par les Britanniques à tous les reporters, Aitken émettait un premier communiqué reproduit par des dizaines de journaux de l'Empire (Gwyn, p. 259). Pour son reportage suivant, qui décrivait la deuxième bataille d'Ypres, en avril 1915, il n'avait probablement pas mis les pieds sur le terrain, mais avait donné des consignes audacieuses à ses rédacteurs: mentionner les noms d'officiers canadiens. Il enfreignit ainsi les règles de la censure afin d'accentuer l'effet d'identification. Ces louanges étaient cependant très sélectives: il semble bien qu'il écrivit et fit écrire de nombreux articles diffusés dans les journaux anglais et européens pour discréditer les Canadiens français ${ }^{7}$.

Il fut ensuite nommé en mai 1915 "War Record Officer", c'est-à-dire archiviste de la guerre avec pour mission de rendre compte des activités de la première division canadienne, puis des trois autres divisions à mesure qu'elles arrivèrent sur le front européen. Il investit son propre argent pour fournir ce que le gouvernement ne payait pas, embaucha à ses frais du personnel 
qu'il logea un temps dans ses propres bureaux à Londres (3, Lombard Street) et paya les frais de voyage de correspondants en France. Pour élargir et consolider cette "entreprise d'information", le Canadian War Records Office (CWRO) fut formé par le gouvernement canadien en janvier 1916 et placé sous la responsabilité d'Aitken avec un budget de 25000 \$, une somme plutôt modeste mais que le directeur saurait bien faire fructifier.

Doté de beaucoup d'argent et de ressources pour représenter et raconter la guerre, le Canadian War Records Office publia des journaux et des livres, tourna des films, organisa des expositions et des conférences, déploya une activité de propagande intense. Aitken embaucha des journalistes pour rédiger les communiqués dont il s'était d'abord chargé, puis les assembla et en tira le livre Canada in Flanders, qui connut un grand succès. Il fonda même un quotidien pour les soldats, le Canadian Daily Record; distribué dans les tranchées, ce journal résumait pour les militaires l'actualité canadienne. Aitken fit embaucher plusieurs des meilleurs peintres canadiens de l'époque (Arthur Lismer, Maurice Cullen, etc.) et les fit voyager sur le front pour qu'ils peignent des tableaux de guerre dont l'exposition et la vente financeraient les secours militaires (hôpitaux, loisirs, etc.). Aitken consacra beaucoup d'énergie à la direction de ces organes, car pour lui le travail d'opinion était indispensable au travail politique: "Publicity for Canada during this war is inseparably bound up with Canada's credit ", écrivait-il au premier ministre Borden (cité dans Chisholm et Davie, p. 127).

Selon l'écrivain Henry Beckles Willson, embauché par Aitken pour organiser la section historique qui compilera les rapports reçus du front, les bureaux de Londres du CWRO étaient en quelque sorte la direction officieuse mais effective du Corps expéditionnaire canadien en Europe (Gwyn, p. 211 et 519). L'efficacité de sa propagande était pour ainsi dire unanimement reconnue. Les alliés enviaient la visibilité qu'il avait procurée aux Canadiens, et même l'ennemi craignait l'efficacité de son appareil d'information. Dans ses mémoires, le généralissime allemand von Ludendorff confie que ses concitoyens étaient "[...] hypnotisés par la propagande ennemie comme un lapin par un serpent. Elle était exceptionnellement brillante et conçue 
sur une grande échelle. Elle reposait sur la persuasion de la masse, informée en détail sur la situation militaire, et utilisait sans scrupule tous les moyens de diffusion" (cité dans Howard, p. 13-14).

Aitken finit par devenir ministre de l'Information de l'Angleterre, le 10 février 1918, grand responsable donc de ce nouveau ministère qu'il avait peut-être encouragé. Mais ses adversaires trouvaient probablement qu'il voulait et prenait trop de place: son mandat recoupait celui du War Propaganda Bureau, créé en 1914 mais demeuré dans l'ombre, et peu enclin à accepter une autorité imposée du dehors. Plusieurs politiciens acceptaient mal que ce poste soit attribué à un important patron de presse. Aitken exerça cette fonction pendant quelques mois, puis prit prétexte d'une maladie pour démissionner élégamment et quitter un terrain mouvant, comme il l'avait fait en délaissant le domaine du ciment canadien... Il ne délaissa pas pour autant le cinéma. Il achète d'abord les réseaux Provincial Cinematograph Theaters et Associated Provincial Picture Houses qui possédaient 80 salles de cinéma anglaises en 1919; peu après, en février 1920, il achète un important bloc d'actions de la firme française Pathé (Low, p. 44). Son objectif fut sans doute de créer un grand réseau de salles et de distribution, mais le cinéma britannique était déjà dominé par les États-Unis. Les timides politiques de quota n'y changèrent pas grand-chose. Selon ses biographes, Aitken rêva même d'unir des services de nouvelles imprimées et filmées, mais les déboires de l'industrie anglaise du film le firent décrocher de ce secteur en 1928 (Chisholm et Davie, p. 255). Il est significatif qu'à cet égard Aitken agisse comme la plupart des capitalistes canadiens, plaçant ses deniers dans la distribution ou l'exploitation, mais hésitant à investir dans la production.

Son court passage au ministère de l'Information le poussa cependant à écrire quelques lignes révélatrices de sa philosophie de l'information et de la propagande:

The function of propaganda is the formation of public opinion. The method is to tell the truth but to present it in an acceptable form. [...] The principle of propaganda is the story of its practice. [...] The 
Ministry or Information is the Ministry of publicity abroad. Its business is to study popular opinion abroad and intluence it through all possible channels, of which the chief is the overseas press. Its object is to state the British case to the World (cité dans Chisholm et Davie, p. 158).

Le représentant canadien qui misait surtout sur le cinéma pour influencer l'opinion britannique est devenu le représentant britannique tablant sur les journaux pour orchestrer une publicité internationale. C'est qu'entre-temps, déçu de la politique et des politiciens, il s'est tourné vers le développement de ses entreprises de presse. Mais ses théories restent les mêmes d'un secteur à l'autre: influencer l'opinion publique par tous les moyens, imposer les objectifs politiques impériaux britanniques comme seule vérité et fonder la théorie de l'information sur l'efficacité persuasive.

Airken disposait d'un personnel considérable; il recevait de l'aide pour recruter de bons employés ainsi que suffisamment d'argent pour bien les payer. Il embaucha donc aussi des cameramen. Comme d'autres dirigeants des pays en guerre, peut-être plus encore qu'eux, il était profondément convaincu de la puissance de l'image animée et jamais il ne cessa de s'en faire le zélateur. Il avait même fait aménager un cinéma à sa maison de campagne où tous les weekends il se faisait projeter les films récents qui l'intéressaient. Cet extrait d'un rapport annuel du CWRO, écrit ou dicté et supervisé par lui, suffira à montrer sa conviction :

But all this labour is well expended, for in the work of publicity the cinematograph stands alone. It creates its own audience without effort, because men are naturally drawn to what they wish to see. And it does so because it contains that subtile mixture of art, reality, and swift and dramatic movement, which rivet the eyes and mind past all withdrawing. [...] The film, then, possesses all those qualities which make it not only an art in itself, but the most powerful instrument for appealing to the mass of the people, and the Dominion Government has been the first to realise the potency of the new iveapon... (Aitken, p. 6). 
Aitken pense que le film est une arme, que sa puissance repose sur sa capacité de subjuguer son spectateur, que l'État doit donc l'utiliser au maximum. Ses convictions rejoignent les professions de foi que feront Dziga Vertov et John Grierson une ou deux décennies plus tard. Et comme eux, Aitken obtint la latitude nécessaire pour passer de la théorie à la pratique; plus, il obtint probablement davantage de latitude et d'argent qu'aucun de ses successeurs. À tel point que tout le corpus des films de guerre produits par l'Angleterre et les pays alliés pourrait être présenté comme son œuvre : il en commanda et en suivit de près l'exécution, il donna même des directives afin d'assurer sa conservation après la guerre. La production canadienne, ou relative aux opérations canadiennes, remise au gouvernement après la guerre et restée introuvable jusqu’à récemment, atteignit environ 15000 mètres de pellicule, chiffre considérable pour l'époque et le pays. Chaque bataille importante, impliquant les forces canadiennes, ou à tout le moins les opérations préparatoires et postérieures aux affrontements, fut filmée. Les opérateurs ne pouvaient montrer les combats, mais tous les autres aspects de l'intervention furent abondamment filmés par les cameramen affectés en permanence aux opérations canadiennes entre 1915 et 1919: recrutement au Canada, entraînement en Angleterre, déplacements des troupes, vie dans les tranchées, soins aux blessés, opérations de support et d'intendance, défilés des troupes victorieuses ou des prisonniers capturés, etc. Ces films étaient diffusés régulièrement en Angleterre et au Canada avec les newsreels relatifs à la guerre, mais aussi montés en séries thématiques illustrant les batailles importantes ou les différents aspects des opérations militaires. Ramenés au Canada en 1919, les films furent utilisés pour la production du film commémoratif Lest We Forget produit en 1935; les négatifs furent détruits dans l'incendie de l'entrepôt de l'Office national du film en 1967, mais des copies ont été retrouvées par l'auteur du présent article dans le fonds d'archives commerciales (stock shot) de l'ONF, qui veut maintenant entreprendre la reconstitution de la collection originale ${ }^{8}$.

Le triste sort de ces archives dispersées et négligées est assez paradoxal, puisque Aitken voyait son travail comme celui d'un 
propagandiste, mais aussi d'un historien. C'est cette perspective à long terme qui justifie sa vision biaisée de la réalité militaire. Pendant que les soldats défendaient fébrilement les dernières heures de leur vie, cet homme pensait plutôt à la fonction historique de leurs souffrances et aux générations futures qui pourront juger de leur conservation: "Posterity must be the arbiter. [...] The Office has collected the materials of the history of the future. [...] The aim of the office has not been to supply an essay for the moment, but a possession for all time" (Aitken, p. 2). Pour lui, les images n'étaient pas destinées aux citoyens de cette époque, ni semble-t-il aux soldats qui se battaient sur le terrain, mais plutôt à leurs concitoyens restés au pays. Aitken croyait en effet que les Canadiens désiraient connaître en détail la participation des leurs aux opérations militaires et il justifiait cette intense couverture en déclarant que la démocratie est fondée sur la connaissance et la raison:

From every county in Canada men have flocked to join the flag. The record of every localiry is being compiled, and in the case of the earlier units is already complete. [...] We must acknowledge if we are to have light and withour light nothing great is accomplished, for it is impossible to ask a free democracy to sacrifice itself without knowledge and reason. It is the insurgence of freedom breaking through the trammels which tradition has imposed on it which will break the Prussians; otherwise, there is no hope (p. 4).

Aitken avait pour mission de rendre compte des activités des armées canadiennes en Europe. Il ne le fit jamais sans intégrer les opérations à un discours sur la défense de la démocratie britannique contre la barbarie germanique. Par exemple, à propos de la première division, il écrivit dans son premier rapport: "That Division stood at Ypres in May, 1915, at the last bulwark of civilisation against the triumphant inroads of barbarism." Sa propre définition de la démocratie s'accommodait cependant assez bien de la censure et d'une conception de l'information limitée à la propagande. Selon cette logique, en temps de guerre, la démocratie doit être atténuée et maintenue sous haute surveillance afin d'être protégée. La lecture libre 
devient une lecture dirigée par des textes soigneusement préparés, composés, distribués. Les opérateurs embauchés par Aitken répètent son discours presque mot à mot et appliquent rigoureusement les consignes qui en découlent, comme on peut le constater en lisant le livre que le cameraman Geoffrey Malins a écrit sur son expérience pendant les deux premières années de la guerre: "To me has been entrusted the task of securing for the enlightenment and education of the people of today, and of future generations, such a picture as will stir their imagination and thrill their hearts with pride» (Malins, p. 4).

Une grande partie du public lecteur souscrira à cette lecture enrégimentée. Le Canada fit un immense effort de guerre, un des plus importants proportionnellement à sa population: le pays avait mobilisé 595000 hommes, dont 418000 avaient traversé en Europe et 21000 autres avaient été incorporés à l'armée britannique. En comptant les marins et les infirmières, le total des mobilisés atteint 619000 , ce qui est énorme pour une petite population comme celle du Canada ( 8 millions d'habitants en 1914). Ces chiffres représentent une proportion d'environ un homme sur cinq, donc presque tous les Canadiens en état de porter les armes, considérant que très peu de Canadiens français s'enrôlèrent et que les femmes, les enfants et les personnes âgées étaient évidemment exclus. Leffort économique fut aussi phénoménal. Le Canada dépensait 15 millions de dollars pour sa défense en 1913, mais il y consacra 311 millions en 1916, instaurant pour y parvenir l'impôt sur le revenu et les emprunts de la victoire (Morton et Granatstein, p. 85). Ces chiffres montrent l'ampleur des moyens déployés. Limportant effort pour produire des films visait à accentuer la propagande pour la mobilisation, mais sans doute aussi à montrer aux pays alliés (dont une partie de ces films circulait sur leur territoire) l'importance de l'effort canadien. L'autorité canadienne envoyait ses soldats se battre en Europe autant pour une place symbolique à la table des nations que pour défendre du terrain sur le front européen ou lutter contre la "barbarie germanique». Les films devaient surtout convaincre les Canadiens de la nécessité de la guerre et les étrangers de l'importance de la participation canadienne. La guerre 1914-1918 a permis au Canada de 
s'asseoir à la même table que des nations dix fois plus puissantes, en reconnaissance d'efforts civils et militaires disproportionnés. Les films de guerre ne peuvent être lus et compris sans mentionner cet effort gigantesque et l'intense propagande qu'il nécessitait. Dans ce contexte, on comprend mieux l'importante production cinématographique canadienne pendant cette guerre et le pouvoir illimité concédé à son responsable.

\section{L'après-guerre et l'après-lecture}

À ce stade-ci, une question majeure s'impose: jusqu'à quel point la politique cinématographique mise en œuvre par William Maxwell Aitken pour le compte du gouvernement canadien peut-elle avoir préparé celle du Britannique John Grierson arrivé au Canada en 1939? Des similitudes sont évidentes: la primauté du documentaire, une production centralisée dont les francophones sont presque absents, une lecture triomphaliste et évidemment partisane de la guerre, reconnue comme telle par une bonne partie du public canadien, et dès lors, non destinée à le convaincre mais plutôt à stimuler son ardeur en évitant de montrer la mort, sinon celle de l'ennemi. Pendant la Deuxième Guerre mondiale, le peuple canadien mourait sur les champs de bataille, comme à Ypres, Vimy et Paschendaele en 1914; le gouvernement canadien, quant à lui, en sortit triomphant. La lutte contre le nazisme en 1939 justifiait certainement une guerre, mais le cinéma enrôlé est un cinéma de mort, tout comme un conscrit est un soldat mort, et la démocratie qui a vaincu les hordes hitlériennes est une démocratie infirme, amputée et boiteuse, blessée par trop de petites et grandes guerres. Si les Allemands avaient pu voir de vrais films de guerre, ils ne se seraient peut-être pas enrôlés; si les Canadiens avaient vu de vrais films de guerre, leurs dirigeants n'auraient peut-être pas eu à décréter la conscription. Mais la lecture libre est-elle possible dans des sociétés prisonnières de l'État et de l'oligarchie financière? Et cette question, peut-on même la poser?

Si le corps des films produits sous la direction de Grierson entretient des liens de parenté étroits avec celui constitué sous Aitken, c'est que les positions et les décisions prises par ce 
dernier ont pesé fortement sur l'histoire du cinéma canadien. La création du Canadian Government Motion Picture Bureau après la guerre, de même que l'orientation nationaliste canadienne des firmes privées comme Pathéscope of Canada et British Canadian Pathé News (Ouimet) et le soutien que ces compagnies obtinrent du gouvernement canadien sont de toute évidence la suite logique de l'orientation prise durant la Première Guerre mondiale. Certains documents soutiennent éloquemment cette hypothèse. Ainsi, une lettre du président du Conseil privé à Edward Kemp, ministre canadien des Forces armées du Canada outremer, informe ce dernier que Pathéscope a signé un contrat avec le gouvernement pour produire des films d'actualité canadienne capables de supplanter les newsreels américains. Il demande à Kemp de collaborer en l'informant des opérations de rapatriement et de démobilisation pouvant être filmées par Pathéscope: "This important experiment which should be of real value in the development of Canadian national sentiment can only be made a success by the hearty cooperation of all departments of the government". "Cette lettre montre que même si l'autorité politique consent à confier la production aux firmes privées après la guerre, elle tient néanmoins à ce que le sentiment national soit une préoccupation essentielle. La série de films d'actualité produite pendant deux ans (1919-1921) par Pathéscope fut d'ailleurs intitulée Canadian National Pictorial. La lettre du Conseil privé laisse aussi deviner une importante activité de lobbying de la part des producteurs de films, conscients de l'importance du sentiment national pour les politiciens, mais bien naïfs quant à la volonté du gouvernement de soutenir une vraie industrie nationale du cinéma.

L'étude de l'organisation de la propagande britannique et de son évolution montre qu'on mit beaucoup de temps à admettre l'importance du cinéma en Angleterre; elle révèle aussi que Max Aitken jouissait d'une bien plus grande liberté du côté canadien et que le succès de sa propagande influença sans doute fortement les décisions anglaises. Mais alors que "Sir Max» semblait agir selon son bon vouloir, les décisions anglaises devaient subir l'épreuve de la concertation. La propagande filmée anglaise, disparate et produite par plusieurs ministères, ne devint réellement 
structurée et efficace qu'en 1917, après que le premier ministre libéral Asquith eut dû céder le pouvoir à David Lloyd George (en décembre 1916), qui réorganisa la propagande en formant un Département de l'information qui deviendrait en 1918 le ministère de l'Information confié à Aitken. Le premier ministre lui confia cette responsabilité surtout à cause de sa place déjà majeure dans les journaux, qu'on voyait comme le principal moyen d'une grande offensive d'information. Il semble que le besoin était devenu pressant pour l'autorité politique de stimuler les travailleurs anglais scandalisés par les profits que les industriels tiraient de la guerre (Evans, p. 20). La compétence d'Aitken comme propagandiste étant évidente et reconnue, il allait presque de soi qu'il finisse par assumer la direction de la propagande anglaise en plus de celle du Canada. Mais les Britanniques ne lui permettront jamais d'exercer le contrôle qu'il pouvait avoir au Canada. La rivalité entre les différents ministères anglais et leurs chefs ne cessa pas et jamais la propagande n'eut une direction unifiée, une étude officielle suggérant d'ailleurs de former un organisme central pour transiger avec l'industrie du film, plutôt que pour unifier la production gouvernementale (Evans, p. 23).

D'autre part, il semble que la latitude laissée à Lord Beaverbrook par le Canada visait l'exclusion de l'entreprise privée afin que les profits du cinéma aillent entièrement aux secours militaires. Cela indiquerait une importante responsabilité de l'autorité militaire canadienne dans la production cinématographique, ou du moins une considération beaucoup plus grande des fins militaires et politiques que des besoins de l'industrie cinématographique. L'état rudimentaire de cette industrie au Canada explique sans doute cette position; elle explique également le pouvoir acquis par Aitken et la politique du contrôle total élaborée sous sa direction. La fondation de l'ONF en 1939, alors que la guerre était imminente, montre la continuité de la politique canadienne: on refuse de soutenir l'industrie du film canadien, on flirte avec Hollywood, mais on impose un contrôle totalitaire sur le film documentaire dès que la situation militaire l'exige. À cet égard, le parcours d'Isaac Walton Killam est exemplaire. Autre richissime financier 
canadien qui fut d'abord l'employé puis l'associé et ami d'Aitken de qui il acheta Royal Securities en 1919, Killam deviendra un des propriétaires de Famous Player's Canadian Corporation, firme qui organisa le monopole de la distribution du film hollywoodien au Canada dans les années 1920, monopole si puissant qu'il entraîna une enquête gouvernementale sous la direction du juge White. Les choses sont presque limpides: les magnats canadiens de la finance ne croyaient pas à une industrie canadienne du cinéma et se contentaient de participer aux profits de l'exploitation et de la distribution du film américain, se réservant cependant la main haute sur le film documentaire comme outil de maintien de l'identité nationale canadienne. Celle-ci, au lieu d'être une fiction attrayante et populaire, est un documentaire oublié parce que atrophié.

Université Laval

\section{NOTES}

1 Ce texte a été produit dans le cadre d'une recherche postdoctorale subventionnée par le Conseil de recherches en sciences humaines du Canada. Laureur prépare un ouvrage intitulé Lectures de guerre, traitant de la pragmatique du cinéma de propagande.

2 Des contacts suivis furent d'ailleurs maintenus par Grierson avec ses patrons anglais, comme le montrent les letrres qu'il échangeait avec son ancien patron Tallents (Gary Evans, "John Grierson et la propagande au Québec et au Canada pendant la Deuxième Guerre mondiale ", dans Claude Beauregard et Catherine Saouter (direction), Conflits contemporains et médias, Montréal: XYZ éditeur, 1997, p. 107-108).

3 Fondé sur l'intervention dans les trois champs de la finance, la politique et la propagande, ce programme fut celui d'Aitken pendant toute sa vie, comme le montrent toutes ses biographies.

4 Les vues d'Aitken sur la politique et ses démêlés avec les politiciens anglais sont décrits dans quelques-uns des livres qu'il a écrits ou fait écrire: Politicians and the Press (1925), Politicians and the War vol. I (1928), Politicians and the War vol. 2(1932). Il a aussi publié en 1921 un livre intitulé Success qui est surtout une profession de foi envers l'entreprise privée.

5 Cette amitié prit fin quelques années plus tard lorsque Aitken prit parti pour l'indépendance irlandaise, peut-être surtout parce qu'il admirait le chef irlandais Tim Healy. De la même façon, son support occasionnel pour l'Union soviétique était peutêtre dù à son admiration pour la poigne manipulatrice de Staline.

6 Peter Howard écrit qu'il coordonnait toute la propagande, assisté par Lord Northcliffe pour la propagande vers les pays ennemis, Rudyard Kipling pour la propagande nationale et coloniale, et Lord Rothermere pour les pays neutres 
(Beaverbrook. A Study of Max the Unknown, Londres/Fredericron: Hutchinson/ Brunswick Press, 1964, p. 14).

7 Aitken était détesté par les volontaires canadiens-français, qui connaissaient son rôle dans la diffusion de ces articles. Thomas-Louis Tremblay, commandant du $22^{\circ}$ bataillon, le rencontra par hasard en novembre 1917; il lui conseilla de dire à ses amis de "se la fermer" et "d'éviter le 22 bataillon" (Jean-Pierre Gagnon, Le 22" bataillon (canadien-franf̧ais) 1914-1919. Etude sociv-militaire, Otrawa/Québec: Défense nationale/Presses de l'Université Laval, 1986, p. 266-267).

8 Voir à ce sujet mon article, "Les fìms perdus de la guerre oubliée". Canadian Journal of Film Studies, vol. 7, n" 1 (1998), p. 29-42.

9 Letre du présidenr du Conseil privé à Sir Edward Kemp, 18 juin 1919 (Archives nationales du Canada, série RG-9-III, Vol. 72).

\section{OUVRAGES CITÉS}

Aitken, Max. Canadian War Records Office Report, 30 mars 1918. Archives nationales du Canada, série RG..9 III, vol. 72.

Chambers, Ernest. «Rapport sur le service de la censure de la presse canadienne, Ottawa, 1920». Cahiers d'bistoire politique, n" 2 (1996), p. 185-288.

Chisholm, Anne, et M. Davie. Lord Beaverbrook. A Life. New York: Alfred Knopf, 1993.

Eksteins, Modris. "The Cultural Impact of the Great War", dans Karel Dibbets et Bert Hogenkamp (direction), Film and the First World War. Amsterdam: Amsterdam University Press (1995), p. 201-212.

Evans, Gary. The War for Men's Minds: Government Film Propaganda in Britain and Canada, 1914-1945 (thèse de doctorat). Montréal: Université McGill, 1977.

Evans, Gary, "John Grierson et la propagande au Québec et au Canada pendant la Deuxième Guerre mondiale", dans Claude Beauregard et Catherine Saouter (direction), Conflits contemporains et médias. Montréal : XYZ éditeur, 1997.

Gagnon, Jean-Picrre. Le 22 bataillon (canadien-franģais) 1914-1919. Étude sociomilitaire. Ottawa/Québec: Défense nationale/Presses de l'Université Laval, 1986.

Gwyn, Sandra. Tapestry of War. A Private View of Canadians in the Great War. Toronto: Harper-Collins Publishers, 1992.

Howard, Peter. Beaverbrook. A Study of Max the Unknown. Londres/Fredericton: Hutchinson / Brunswick Press, 1964.

Rachael Low, The History of the British Film 1918-1929. Londres: George Allen \& Unwin, 1973.

Malins, Geoffrey H. How I Filmed the War. Londres: Herbert Jenkins, 1920.

Mc Kernan, Luke. Topical Budget. The Great British News Film. Londres: British Film Institute, 1992.

Morton, Desmond, et J. L. Granatstein. Marching to Armageddon. Canadians and the Great War 1914-1919. Toronto: Lester and Orpen Dennys, 1989.

Odin, Roger. "Film documentaire. Lecture documentarisante", dans R. Odin et J.-C. Lyant (direction), Cinémas et réalités. Saint-Étienne: CIEREC (1984), p. 263-278.

Odin, Roger. «Approche sémio-pragmatique, approche historique. De l'intérêt du dialogue ». Ars Semeiotica, vol. 17, nis 1-4 (1994), p. 27-36.

Véronneau, Pierre. "Le rapport Grierson dans son contexte", John Grierson. Rapport sur les activités cinématographiques du gouvernement canadien. Montréal: Cinémathèque québécoise (1978). 